\title{
Zum thermischen Verhalten einiger Kohlensäure[(methylphenylsilyl)methyl]ester-Derivate
}

On the Thermal Behaviour of Some (Methylphenylsilyl)methyl Carbonate Derivatives

Reinhold Tacke*, Matthias Link, Anke Bentlage-Felten und Harald Zilch

Institut für Anorganische und Analytische Chemie der Technischen Universität Braunschweig, Hagenring 30, D-3300 Braunschweig

Z. Naturforsch. 40b, 942-947 (1985); eingegangen am 16. März 1985

(Methylphenylsilyl)methyl Carbonates, Rearrangement, Dimethylphenylsilyl Carbonates,

Decarboxylation

The synthesis and the thermal behaviour of the (methylphenylsilyl)methyl carbonates $\mathrm{CH}_{3}\left(\mathrm{C}_{6} \mathrm{H}_{5}\right) \mathrm{Si}(\mathrm{H}) \mathrm{CH}_{2} \mathrm{OC}(\mathrm{O}) \mathrm{X}\left(6: \mathrm{X}=\mathrm{OCH}_{3} ; 7: \mathrm{X}=\mathrm{Cl} ; \mathbf{8}: \mathrm{X}=\mathrm{N}\left(\mathrm{CH}_{3}\right)_{2}\right)$ is described. 8 rearranges in toluene solution at $100{ }^{\circ} \mathrm{C}$ quantitatively to give the carbamoyloxysilane $\mathrm{C}_{6} \mathrm{H}_{5}\left(\mathrm{CH}_{3}\right)_{2} \mathrm{SiOC}(\mathrm{O}) \mathrm{N}\left(\mathrm{CH}_{3}\right)_{2}(\mathbf{1 1})$, whereas neat 6 and 7 at $135^{\circ} \mathrm{C}$ undergo quantitative formation of $\mathrm{C}_{6} \mathrm{H}_{5}\left(\mathrm{CH}_{3}\right)_{2} \mathrm{SiOCH}_{3}(\mathbf{1 2})$ and $\mathrm{C}_{6} \mathrm{H}_{5}\left(\mathrm{CH}_{3}\right)_{2} \mathrm{SiCl}(\mathbf{1 3})$, respectively. The formation of 12 and $\mathbf{1 3}$ is explained by a rearrangement reaction (by analogy to the rearrangement of $\mathbf{8}$ ), followed by a decarboxylation. The thermally induced transformations $6 \rightarrow 12,7 \rightarrow 13$, and $8 \rightarrow 11$ were found to be first-order reactions with half-lifes of $\sim 2.6 \mathrm{~h}\left(135^{\circ} \mathrm{C}\right.$, neat $), \sim 4.5 \mathrm{~h}\left(135^{\circ} \mathrm{C}\right.$, neat), and $\sim 3.7 \mathrm{~h}\left(100^{\circ} \mathrm{C}\right.$, in toluene), respectively.

Carbonsäure[(diorganylsilyl)methyl]ester 1 [1] und Trifluormethansulfonsäure[(diorganylsilyl)methyl] ester 3 [2] unterliegen einer thermisch induzierten Umlagerung $\mathrm{zu}$ den entsprechenden (Methyldiorganylsilyl)estern 2 bzw. 4.

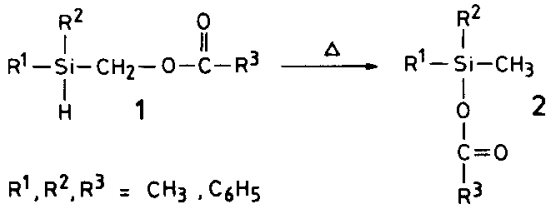

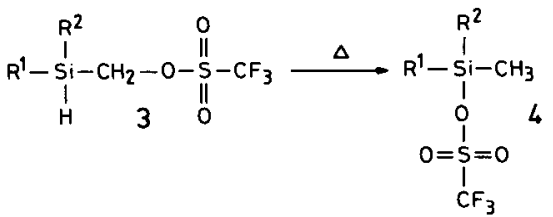

Wir berichten hier über das thermische Verhalten der mit 1 und $\mathbf{3}$ strukturverwandten Kohlensäure[(methylphenylsilyl)methyl]ester 6-8, welche - jeweils ausgehend von (Hydroxymethyl)methylphenylsilan (5) [3] - erstmalig synthetisiert worden sind. Durch Umsetzung von 5 mit Chlorameisensäure-

\footnotetext{
* Sonderdruckanforderungen an Priv.-Doz. Dr. R. Tacke. Verlag der Zeitschrift für Naturforschung, D-7400 Tübingen 0340-5087/85/0700-0942/\$01.00/0
}

methylester in Pyridin (Lösungsmittel und HCl-Akzeptor) wurde der entsprechende Kohlensäurediester 6, durch Umsetzung mit Phosgen das Kohlensäureesterchlorid 7 dargestellt. Letzteres konnte mit Dimethylamin in das entsprechende Kohlensäureesteramid 8 übergeführt werden. Die genannten Verbindungen wurden dabei mit Ausbeuten von 66 (6), 96 (7) bzw. 93\% (8) in Form farbloser, ${ }^{1} \mathrm{H}$ NMR-spektropskopisch reiner Flüssigkeiten erhalten, die sich im Vakuum unter schonenden Bedingungen (vgl. hierzu den exp. Teil) unzersetzt destillieren ließen. Sie wurden in ihrer Struktur durch Elementaranalysen und spektroskopische Untersuchungen abgesichert.

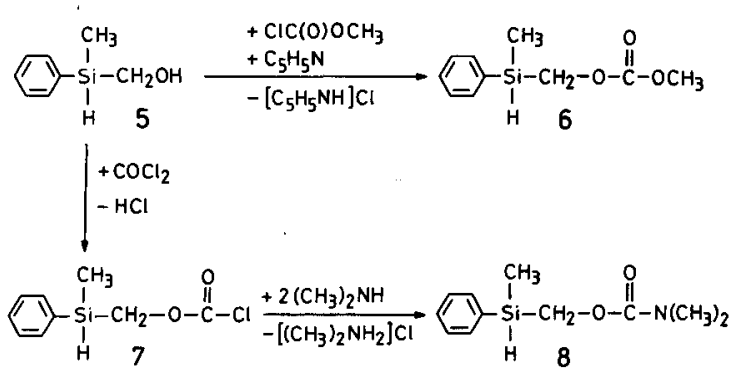

In Analogie zu den Umlagerungen $\mathbf{1} \rightarrow \mathbf{2}$ und $\mathbf{3} \rightarrow \mathbf{4}$ konnte für die Verbindungen 6-8 eine thermisch induzierte Umwandlung in die Silane 9-11 erwartet werden, wie wir dies im Falle des Kohlensäureester- 
amids 8 auch tatsächlich bestätigt fanden: 41-stdg. Erhitzen von 8 in Toluol auf $100^{\circ} \mathrm{C}$ ergab praktisch quantitativ das (Dimethylcarbamoyloxy)silan 11. Die thermische Behandlung des Kohlensäurediesters $\mathbf{6}$ und des Kohlensäureesterchlorids $\mathbf{7}$ führte dagegen zur Bildung des Methoxysilans 12 bzw. des Chlorsilans 13; die potentiellen Umlagerungsprodukte von 6 und 7 - die Verbindungen 9 und 10 - konnten weder isoliert noch als Zwischenprodukte nachgewiesen werden $\left({ }^{1} \mathrm{H}\right.$-NMR-Kontrolle des Reaktionsablaufes). 29-stdg. Erhitzen von 6 und 50-stdg. Erhitzen von 7 auf $135^{\circ} \mathrm{C}-$ jeweils in Substanz durchgeführt - ergab unter $\mathrm{CO}_{2}$-Abspaltung in glatter Reaktion die Silane 12 bzw. 13, welche mit hoher Ausbeute isoliert werden konnten.

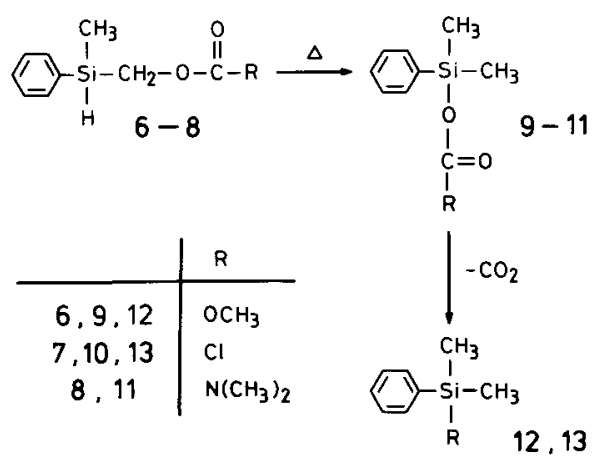

${ }^{1} \mathrm{H}$-NMR-spektroskopische Untersuchungen ergaben, daß die thermisch induzierten Umwandlungen $6 \rightarrow \mathbf{1 2}, 7 \rightarrow \mathbf{1 3}$ und $8 \rightarrow \mathbf{1 1}$ nach einem Geschwindigkeitsgesetz 1. Ordnung ablaufen. Die entsprechenden kinetischen Parameter sind in Tab. I zusammengestellt (vgl. hierzu den exp. Teil). In allen drei Fällen wurde ein eindeutiger Reaktionsablauf beobachtet; Zwischen- bzw. Nebenprodukte konnten ${ }^{1} \mathrm{H}$ NMR-spektroskopisch nicht nachgewiesen werden. Allerdings zeigte sich, daß 8 (im Gegensatz zur glatten Umwandlung von 6 und 7) nur dann einheitlich

Tab. I. Kinetische Parameter der Umwandlungen von 6-8.

\begin{tabular}{llll}
\hline Umwandlung & Temperatur $\left[{ }^{\circ} \mathrm{C}\right]$ & $t_{1 / 2}[\mathrm{~h}]$ & $k\left[10^{-5} \mathrm{~s}^{-1}\right]$ \\
\hline $\mathbf{6} \rightarrow \mathbf{1 2}$ & 135 & 2,6 & 7,3 \\
$\mathbf{7} \rightarrow \mathbf{1 3}$ & 135 & 4,5 & 4,2 \\
$\mathbf{8} \rightarrow \mathbf{1 1}$ & 100 & 3,7 & 5,2
\end{tabular}

${ }^{a}$ Vgl. hierzu den experimentellen Teil. in 11 übergeführt werden kann, wenn die Umlagerung in Lösung erfolgt: Wird $\mathbf{8}$ dagegen in Substanz erhitzt, so beobachtet man neben dem Hauptprodukt 11 zusätzlich noch einige bisher nicht identifizierte Nebenprodukte. Sicher ist jedoch, daß es sich bei letzteren nicht um die von $\mathbf{8}$ und $\mathbf{1 1}$ abzuleitenden potentiellen Decarboxylierungsprodukte 16 bzw. 14 handelt $\left({ }^{1} \mathrm{H}\right.$-NMR-Vergleich mit authentischen Proben von 16 und 14, welche durch Umsetzung von 15 bzw. 13 mit Dimethylamin erhalten wurden).
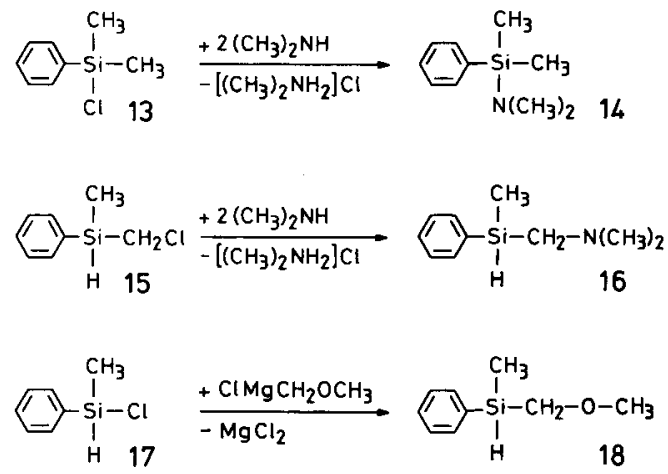

Zur Deutung der Umwandlungen $6 \rightarrow \mathbf{1 2}, 7 \rightarrow \mathbf{1 3}$ und $\mathbf{8} \rightarrow \mathbf{1 1}$ wird als Arbeitshypothese angenommen, daß die Silane 6-8 einheitlich zu den Verbindungen 9-11 umlagern und die beiden Kohlensäure-Derivate 9 und 10 - im Gegensatz zu dem thermisch sehr beständigen Derivat $\mathbf{1 1}$ - anschließend rasch zu $\mathbf{1 2}$ bzw. 13 decarboxylieren; die Umlagerungsschritte $6 \rightarrow 9$ und $7 \rightarrow \mathbf{1 0}$ der formal zweistufigen Umwandlungen $6 \rightarrow 12$ bzw. $7 \rightarrow 13$ sind dabei jeweils geschwindigkeitsbestimmend. Eine umgekehrte Abfolge der beiden Schritte - d.h. zuerst Decarboxylierung und dann Umlagerung - kann ausgeschlossen werden: Das (Methoxymethyl)silan 18 (zugänglich durch. Umsetzung des entsprechenden Chlorsilans 17 mit $\mathrm{ClMgCH}_{2} \mathrm{OCH}_{3}$ ) und das (Chlormethyl)silan 15, welche formal als potentielle Decarboxylierungsprodukte der Kohlensäure-Derivate 6 bzw. 7 aufgefaßt werden können, lagern sich bei thermischer Belastung bis auf $200^{\circ} \mathrm{C}$ erwartungsgemäß nicht in die Verbindungen 12 und 13 um (16 ist unter diesen Bedingungen ebenfalls stabil): Das Silan 18 wurde nach mehrstündigem Erhitzen auf $200{ }^{\circ} \mathrm{C}$ unverändert vorgefunden ( ${ }^{1} \mathrm{H}-\mathrm{NMR}-$ Kontrolle $)$ und scheidet somit als Zwischenprodukt der Umwandlung $\mathbf{6} \rightarrow \mathbf{1 2}$ aus. Verbindung $\mathbf{1 5}$ zeigte unter den glei- 
chen Bedingungen Zersetzungserscheinungen, die sich bereits bei $135^{\circ} \mathrm{C}$ schwach andeuteten; eine Umlagerung in 13 konnte jedoch nicht ausgemacht werden ( ${ }^{1} \mathrm{H}-\mathrm{NMR}-\mathrm{Kontrolle}$ ), weshalb 15 als Zwischenprodukt der Umwandlung $\mathbf{7} \rightarrow \mathbf{1 3}$ nicht in Frage kommt.

Das von 11 abweichende Decarboxylierungsverhalten von 9 und 10 - wie hier zur Deutung des unterschiedlichen thermischen Verhaltens von 6-8 angenommen - steht im Einklang mit Literaturangaben zur Stabilität von Kohlensäuresilylester-Derivaten: Entsprechend Lit. [4] ist es bisher nicht gelungen, (Chlorformyloxy)triorganylsilane des Typs 20 $\mathrm{zu}$ isolieren. Aus der Klasse der (Organyloxycarbonyloxy)triorganylsilane 19 sind dagegen einige oberhalb Raumtemperatur stabile Vertreter bekannt, während andere unter diesen Bedingungen decarboxylieren $[5,6]$; bei höheren Temperaturen $\left(150-200^{\circ} \mathrm{C}\right)$ unterliegen jedoch auch die stabileren Derivate einer merklichen $\mathrm{CO}_{2}$-Abspaltung [5]. (Diorganylcarbamoyloxy)triorganylsilane des Typs 21 sind dagegen im Vergleich zu 19 und 20 - wie auch durch das Beispiel von 11 belegt - recht beständig $[5,7,8]$. Für vereinzelte Vertreter von 21 sind zwar Decarboxylierungstendenzen beschrieben, die sich jedoch erst oberhalb von $180^{\circ} \mathrm{C}$ schwach andeuten [8]. Mehrstündiges Erhitzen von 11 (ohne Lösungsmittel) auf $200^{\circ} \mathrm{C}$ führt zu keiner erkennbaren ( $\left.{ }^{1} \mathrm{H}-\mathrm{NMR}\right)$ Veränderung der Substanz.

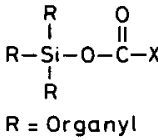

\begin{tabular}{l|l} 
& $x$ \\
\hline 19 & OR \\
20 & $\mathrm{Cl}$ \\
21 & $\mathrm{NR}_{2}$
\end{tabular}

Die hier vorgestellten Untersuchungen zeigen, daß die an [(Diorganylsilyl)methyl] estern von Carbonsäuren [1] und der Trifluormethansulfonsäure [2] beobachteten Umlagerungsreaktionen auch mit entsprechenden Kohlensäureester-Derivaten ablaufen. Es scheint sich hierbei um ein allgemeineres Reaktionsprinzip zu handeln, das offensichtlich für [(Diorganylsilyl)methyllester-Derivate einer ganzen Reihe von Elementsauerstoffsäuren Gültigkeit besitzt. Kürzlich fanden wir, daß auch entsprechende Phosphorsäureester einer analogen Umlagerung unterliegen, wie z. B. an der bei $60^{\circ} \mathrm{C}$ in Benzol ablaufenden Umwandlung von 22 in 23 beobachtet [9]. Diese Reaktion beinhaltet formal zwei Umlagerungsschritte - bedingt durch die Anwesenheit zweier ,umlagerungsfähiger" Struktureinheiten in 22.

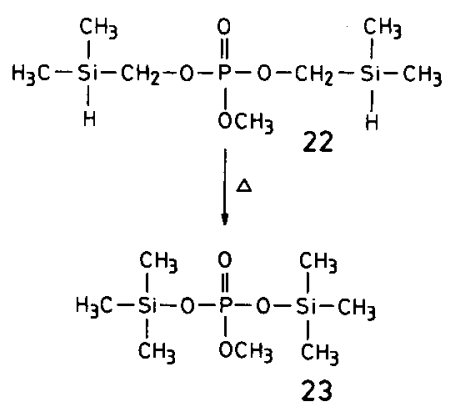

\section{Experimenteller Teil}

Brechungsindices: Abbé-Refraktometer (Fa. Zeiss). - ${ }^{1} \mathrm{H}-\mathrm{NMR}-S p e k t r e n$ (400,1 MHz): WM400-Gerät (Fa. Bruker). - IR-Spektren: AccuLab-9Gerät (Fa. Beckman). - Massenspektren (70 eV; angegeben sind ausgesuchte, strukturbeweisende

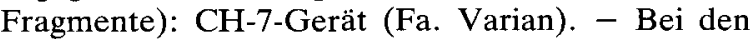
kinetischen Messungen wurde mit einem Bad-Thermostaten D 3-19 (Fa. Haake) eine Temperaturkonstanz von $\pm 0,02{ }^{\circ} \mathrm{C}$ sichergestellt. Die Kinetik wurde ${ }^{1}$ H-NMR-spektroskopisch verfolgt (R-24B-Gerät, $60 \mathrm{MHz}$, Fa. Hitachi-Perkin-Elmer). - Alle Reaktionen wurden unter einer Schutzgasatmosphäre von trockenem Stickstoff durchgeführt; die verwendeten Lösungsmittel waren wasserfrei.

(Hydroxymethyl)methylphenylsilan (5) wurde gemäß Lit. [3] hergestellt.

(Methoxycarbonyloxymethyl)methylphenylsilan (6): $\mathrm{Zu}$ einer Lösung von 10,66 g (70 mmol) 5 in $40 \mathrm{ml}$ Pyridin wurden unter Eiskühlung und starkem Rühren langsam 6,62 g (70 mmol) Chlorameisensäuremethylester getropft. Anschließend rührte man $3 \mathrm{~h}$ bei Raumtemp., versetzte das Reaktionsgemisch mit $50 \mathrm{ml}$ Eiswasser und extrahierte fünfmal mit Ether. Die vereinigten etherischen Extrakte wurden zweimal mit je $40 \mathrm{ml} 6 \mathrm{~N}$ Salzsäure, dann einmal mit $50 \mathrm{ml}$ einer gesättigten wäßrigen $\mathrm{NaHCO}_{3}$-Lösung und schließlich dreimal mit je $50 \mathrm{ml}$ einer gesättigten wäßrigen NaCl-Lösung gewaschen. Nach Trocknen des etherischen Extraktes über $\mathrm{Na}_{2} \mathrm{SO}_{4}$ verdampfte man das Lösungsmittel unter vermindertem Druck und destillierte den Rückstand in Portionen von jeweils $3-4 \mathrm{~g}$ im Kugelrohrverdampfer $\left(90-110^{\circ} \mathrm{C} /\right.$ 0,01 Torr). Ausb. 9,7 g (66\%), $n_{\mathrm{D}}^{20} 1,506 .-{ }^{1} \mathrm{H}-\mathrm{NMR}$ $\left(\mathrm{CCl}_{4}\right): \delta=0,46\left(\mathrm{~d}, J=3,8 \mathrm{~Hz} ; 3 \mathrm{H}, \mathrm{SiCH}_{3}\right), 3,74$ $\left(\mathrm{s} ; 3 \mathrm{H}, \mathrm{OCH}_{3}\right), 4,11\left(\delta_{\mathrm{B}}\right)$ und 4,15 $\left(\delta_{\mathrm{A}}\right)(\mathrm{AB}-\mathrm{Teil}$ des ABX-Systems $\mathrm{Si}\left(\underline{\mathrm{H}}_{\mathrm{X}}\right) \underline{\mathrm{CH}}_{\mathrm{A}} \underline{\mathrm{H}}_{\mathrm{B}} \mathrm{O}, \quad J_{\mathrm{AB}}=$ $-14,3 \mathrm{~Hz}, J_{\mathrm{AX}}=2,4 \mathrm{~Hz}, J_{\mathrm{BX}}=3,2 \mathrm{~Hz} ; 2 \mathrm{H}$, 
$\mathrm{SiCH}_{2} \mathrm{O}$ ), 4,52 (Zentrum eines $\mathrm{m} ; 1 \mathrm{H}, \mathrm{SiH}$ ), 7,3-7,6 (m;5H, $\left.\mathrm{SiC}_{6} \mathrm{H}_{5}\right)$. - IR (Film): 2135 $(\mathrm{Si}-\mathrm{H}), 1750 \mathrm{~cm}^{-1}(\mathrm{C}=\mathrm{O})$. $-\mathrm{MS}: \mathrm{m} / \mathrm{e}=209(47 \%$, $\left.\mathrm{M}^{+}-\mathrm{H}\right), 121\left(100 \%, \mathrm{M}^{+}-\mathrm{CH}_{2} \mathrm{OC}(\mathrm{O}) \mathrm{OCH}_{3}\right)$.

$\mathrm{C}_{10} \mathrm{H}_{14} \mathrm{O}_{3} \mathrm{Si}(210,3)$

$\begin{array}{llll}\text { Ber. } & \text { C } 57,11 & \text { H } 6,71 & \text { Si } 13,35 \text {, } \\ \text { Gef. C } 57,0 & \text { H } 6,8 & \text { Si } 13,5 .\end{array}$

(Chlorformyloxymethyl)methylphenylsilan (7): In $100 \mathrm{ml}$ Toluol wurden bei $0{ }^{\circ} \mathrm{C} 20 \mathrm{~g}(0,2 \mathrm{~mol})$ Phosgen eingeleitet. $\mathrm{Zu}$ dieser Lösung tropfte man bei $-40{ }^{\circ} \mathrm{C}$ unter starkem Rühren innerhalb von $30 \mathrm{~min}$ eine Lösung von $15,23 \mathrm{~g}(0,10 \mathrm{~mol}) \mathbf{5}$ in $30 \mathrm{ml}$ Toluol, ließ unter Rühren auf Raumtemp. erwärmen und rührte dann 30 min weiter. Nachdem man die leichtflüchtigen Bestandteile i. Vak. verdampft hatte, wurde der Rückstand - aufgeteilt in Portionen von jeweils $3-4 \mathrm{~g}-$ im Kugelrohrverdampfer destilliert $\left(65-85^{\circ} \mathrm{C} / 0,01\right.$ Torr $)$. Ausb. 20,6 g $(96 \%), n_{\mathrm{D}}^{20} 1,516 .-{ }^{1} \mathrm{H}-\mathrm{NMR}\left(\mathrm{CDCl}_{3}\right): \delta=0,50(\mathrm{~d}$, $\left.J=3,8 \mathrm{~Hz} ; 3 \mathrm{H}, \mathrm{SiCH}_{3}\right), 4,32\left(\delta_{\mathrm{B}}\right)$ und $4,35\left(\delta_{\mathrm{A}}\right)$ (AB-Teil des ABX-Systems $\mathrm{Si}\left(\underline{\mathrm{H}}_{\mathrm{X}}\right) \mathrm{C}_{\mathrm{H}} \underline{\mathrm{H}}_{\mathrm{B}} \mathrm{O}, J_{\mathrm{AB}}=$ $\left.-14,1 \mathrm{~Hz}, J_{\mathrm{AX}}=2,4 \mathrm{~Hz}, J_{\mathrm{BX}}=3,2 \mathrm{~Hz} ; 2 \mathrm{H}, \mathrm{SiCH}_{2} \mathrm{O}\right)$, $4,55$ (Zentrum eines $\mathrm{m} ; 1 \mathrm{H}, \mathrm{SiH}), 7,2-7,6(\mathrm{~m} ; 5 \mathrm{H}$, $\left.\mathrm{SiC}_{6} \mathrm{H}_{5}\right)$. - IR (Film): $2140(\mathrm{Si}-\mathrm{H}), 1770 \mathrm{~cm}^{-1}$ $(\mathrm{C}=\mathrm{O}) .-\mathrm{MS}: m / e=215 / 213\left(6 \% / 17 \%, \mathrm{M}^{+}-\mathrm{H}\right)$, $121\left(100 \%, \mathrm{M}^{+}-\mathrm{CH}_{2} \mathrm{OC}(\mathrm{O}) \mathrm{Cl}\right)$.

\section{$\mathrm{C}_{9} \mathrm{H}_{11} \mathrm{ClO}_{2} \mathrm{Si}(214,7)$}

$\begin{array}{llll}\text { Ber. } & \text { C } 50,34 & \text { H 5,16 } & \text { Si } 13,08 \text {, } \\ \text { Gef. } & \text { C 50,1 } & \text { H 5,3 } & \text { Si } 13,4 .\end{array}$

(Dimethylcarbamoyloxymethyl)methylphenylsilan (8): Eine Lösung von $10,7 \mathrm{~g}(50 \mathrm{mmol}) 7 \mathrm{in} 30 \mathrm{ml}$ Ether wurde unter Eiskühlung und starkem Rühren innerhalb von $20 \mathrm{~min} \mathrm{zu}$ einer Lösung von $22 \mathrm{~g}$ $(0,49 \mathrm{~mol})$ Dimethylamin in $200 \mathrm{ml}$ Ether getropft. Man rührte noch $1 \mathrm{~h}$ bei $0^{\circ} \mathrm{C}$, filtrierte, befreite das Filtrat i. Vak. von den leichtflüchtigen Anteilen und destillierte den Rückstand - aufgeteilt in Portionen von jeweils 3-4 g - im Kugelrohrverdampfer $\left(80-110^{\circ} \mathrm{C} / 0,01\right.$ Torr). Ausb. 10,3 g (93\%), $n_{\mathrm{D}}^{20}$ 1,516. $-{ }^{1} \mathrm{H}-\mathrm{NMR}\left(\mathrm{CCl}_{4}\right): \delta=0,44$ (d, $J=$ $3,8 \mathrm{~Hz} ; 3 \mathrm{H}, \mathrm{SiCH}_{3}$ ), 2,87 (s, Verbreiterung durch behinderte Rotation, vgl. hierzu Lit. [10]; $6 \mathrm{H}$, $\left.\mathrm{NCH}_{3}\right), 4,01\left(\delta_{\mathrm{B}}\right)$ und 4,03 $\left(\delta_{\mathrm{A}}\right)(\mathrm{AB}-\mathrm{Teil}$ des ABXSystems $\mathrm{Si}\left(\underline{\mathrm{H}}_{\mathrm{X}}\right) \mathrm{CH}_{\mathrm{A}} \underline{\mathrm{H}}_{\mathrm{B}} \mathrm{O}, J_{\mathrm{AB}}=-14,3 \mathrm{~Hz}, J_{\mathrm{AX}}=$ $2,6 \mathrm{~Hz}, J_{\mathrm{BX}}=3,0 \mathrm{~Hz} ; 2 \mathrm{H}, \mathrm{SiCH}_{2} \mathrm{O}$ ), 4,52 (Zentrum eines $\mathrm{m} ; 1 \mathrm{H}, \mathrm{SiH}), 7,3-7,6\left(\mathrm{~m} ; 5 \mathrm{H}, \mathrm{SiC}_{6} \mathrm{H}_{5}\right)$. - IR (Film): $2125(\mathrm{Si}-\mathrm{H}), 1700 \mathrm{~cm}^{-1}(\mathrm{C}=\mathrm{O})$. MS: $m / e=222\left(78 \%, \mathrm{M}^{+}-\mathrm{H}\right), 146 \quad(100 \%$, $\left.\mathrm{M}^{+}-\mathrm{C}_{6} \mathrm{H}_{5}\right), 121\left(100 \%, \mathrm{M}^{+}-\mathrm{CH}_{2} \mathrm{OC}(\mathrm{O}) \mathrm{N}\left(\mathrm{CH}_{3}\right)_{2}\right)$, $72\left(100 \%, \mathrm{M}^{+}-\mathrm{OCH}_{2} \mathrm{Si}(\mathrm{H})\left(\mathrm{CH}_{3}\right) \mathrm{C}_{6} \mathrm{H}_{5}\right)$.

$\mathrm{C}_{11} \mathrm{H}_{17} \mathrm{NO}_{2} \mathrm{Si}(223,3)$
Ber. C 59,16
Gef. C 59,0
$\mathrm{H} 7,67 \mathrm{~N} 6,27$
Si 12,57 ,
H 7,6 N 6,2 Si 12,8.

(Dimethylcarbamoyloxy) dimethylphenylsilan (11): Eine Lösung von $2,00 \mathrm{~g}(8,95 \mathrm{mmol}) \mathbf{8}$ in $20 \mathrm{ml}$ Toluol wurde unter Rühren $41 \mathrm{~h}$ auf $100{ }^{\circ} \mathrm{C}$ erhitzt. Danach entfernte man das Lösungsmittel unter vermindertem Druck und destillierte den Rückstand im Kugelrohrverdampfer $\left(60^{\circ} \mathrm{C} / 0,01\right.$ Torr $)$. Ausb. $1,9 \mathrm{~g}$ $(95 \%)$ einer farblosen Flüssigkeit, die unterhalb $0{ }^{\circ} \mathrm{C}$ fest wird und bei Raumtemp. bereits schmilzt. - ${ }^{1} \mathrm{H}$ NMR $\left(\mathrm{CCl}_{4}\right): \delta=0,41\left(\mathrm{~s} ; 6 \mathrm{H}, \mathrm{SiCH}_{3}\right), 2,66(\mathrm{~s}$, Verbreiterung durch behinderte Rotation, vgl. hierzu Lit. $\left.[11,12] ; 6 \mathrm{H}, \mathrm{NCH}_{3}\right), 7,1-7,5\left(\mathrm{~m} ; 5 \mathrm{H}, \mathrm{SiC}_{6} \mathrm{H}_{5}\right)$. - IR (Film): $1685 \mathrm{~cm}^{-1}(\mathrm{C}=\mathrm{O})$.

\section{$\mathrm{C}_{11} \mathrm{H}_{17} \mathrm{NO}_{2} \mathrm{Si}(223,3)$ \\ Ber. C 59,16 H 7,67 N 6,27, \\ Gef. C 59,4 H 7,7 N 6,3.}

Methoxydimethylphenylsilan (12): $2,00 \mathrm{~g}(9,51 \mathrm{mmol})$ 6 wurden unter Rühren $29 \mathrm{~h}$ auf $135^{\circ} \mathrm{C}$ erhitzt. Die sich anschließende Kugelrohrdestillation (90 bis $110^{\circ} \mathrm{C} / 9$ Torr) lieferte $1,4 \mathrm{~g}(89 \%)$ einer farblosen Flüssigkeit, die in ihren Eigenschaften mit denen einer authentischen Probe von $\mathbf{1 2}$ übereinstimmte, welche durch Methanolyse von $\mathbf{1 3}$ in Gegenwart von Triethylamin erhalten wurde. $-{ }^{1} \mathrm{H}-\mathrm{NMR}\left(\mathrm{CDCl}_{3}\right)$ : $\delta=0,38\left(\mathrm{~s} ; 6 \mathrm{H}, \mathrm{SiCH}_{3}\right), 3,44\left(\mathrm{~s} ; 3 \mathrm{H}, \mathrm{OCH}_{3}\right)$, 7,3-7,6 (m;5 $\left.\mathrm{H}, \mathrm{SiC}_{6} \mathrm{H}_{5}\right)$.

Chlordimethylphenylsilan (13): $2,00 \mathrm{~g}(9,31 \mathrm{mmol})$ 7 wurden unter Rühren 50 h auf $135^{\circ} \mathrm{C}$ erhitzt. Die sich anschließende Kugelrohrdestillation ( 80 bis $110^{\circ} \mathrm{C} / 18$ Torr) lieferte $1,5 \mathrm{~g}(94 \%)$ einer farblosen Flüssigkeit, die in ihren Eigenschaften mit denen einer authentischen Probe von 13 (Handelsprodukt der Fa. Fluka) übereinstimmte. - ${ }^{1} \mathrm{H}$-NMR $\left(\mathrm{CDCl}_{3}\right)$ : $\delta=0,72\left(\mathrm{~s} ; 6 \mathrm{H}, \mathrm{SiCH}_{3}\right), 7,4-7,7\left(\mathrm{~m} ; 5 \mathrm{H}, \mathrm{SiC}_{6} \mathrm{H}_{5}\right)$.

(Dimethylamino) dimethylphenylsilan (14) wurde nach Standardmethoden durch Umsetzung von $\mathbf{1 3}$ mit Dimethylamin in Petrolether hergestellt. $-{ }^{1} \mathrm{H}$ $\operatorname{NMR}\left(\mathrm{CDCl}_{3}\right): \delta=0,50\left(\mathrm{~s} ; 6 \mathrm{H}, \mathrm{SiCH}_{3}\right), 2,68(\mathrm{~s} ; 6 \mathrm{H}$, $\left.\mathrm{NCH}_{3}\right), 7,5-7,7\left(\mathrm{~m} ; 5 \mathrm{H}, \mathrm{SiC}_{6} \mathrm{H}_{5}\right)$.

(Chlormethyl)methylphenylsilan (15) wurde gemäß Lit. [13] hergestellt.

[(Dimethylamino)methyl]methylphenylsilan (16): Eine Mischung aus $6,00 \mathrm{~g}(35,1 \mathrm{mmol}) 15$ und $16 \mathrm{~g}$ $(0,35 \mathrm{~mol})$ Dimethylamin wurde im Einschlußrohr $15 \mathrm{~h}$ auf $80^{\circ} \mathrm{C}$ erhitzt. Anschließend ließ man das überschüssige Dimethylamin verdampfen, versetzte mit $200 \mathrm{ml}$ Petrolether $\left(40-65^{\circ} \mathrm{C}\right)$, filtrierte, befreite das Filtrat unter vermindertem Druck vom Lösungsmittel und destillierte den Rückstand fraktionierend i. Vak. Ausb. $5,7 \mathrm{~g}(90 \%)$, Sdp. $45^{\circ} \mathrm{C} /$ 0,7 Torr, $n_{\mathrm{D}}^{20} 1,509 .-{ }^{1} \mathrm{H}-\mathrm{NMR}\left(\mathrm{CDCl}_{3}\right): \delta=0,40$ (d, $J=3,8 \mathrm{~Hz} ; 3 \mathrm{H}, \mathrm{SiCH}_{3}$ ), 2,18 (,t" ABX-Systems $\mathrm{Si}\left(\underline{\mathrm{H}}_{\mathrm{X}}\right) \mathrm{CH}_{\mathrm{A}} \underline{\mathrm{H}}_{\mathrm{B}} \mathrm{N}$, nicht analysierbar; $2 \mathrm{H}, \mathrm{SiCH}_{2} \mathrm{~N}$ ), 2,27 (s; $\left.6 \mathrm{H}, \mathrm{NCH}_{3}\right), 4,48$ (Zentrum eines $\mathrm{m} ; 1 \mathrm{H}, \mathrm{SiH}), 7,3-7,6\left(\mathrm{~m} ; 5 \mathrm{H}, \mathrm{SiC}_{6} \mathrm{H}_{5}\right)$. - IR 
(Film): $2120 \mathrm{~cm}^{-1}(\mathrm{Si}-\mathrm{H}) .-\mathrm{MS}: m / e=179(39 \%$, $\left.\mathrm{M}^{+}\right), 58\left(100 \%, \mathrm{CH}_{2}=\mathrm{N}\left(\mathrm{CH}_{3}\right)_{2}{ }^{+}\right)$.

\section{$\mathrm{C}_{10} \mathrm{H}_{17} \mathrm{NSi}(179,3)$ \\ Ber. C 66,97 H 9,55 N 7,81 Si 15,66, \\ Gef. C 67,0 H 9,5 N 7,9 Si 16,2.}

Chlormethylphenylsilan (17) wurde in Anlehnung an Lit. [14] durch Umsetzung von Dichlormethylsilan mit Phenylmagnesiumchlorid hergestellt.

(Methoxymethyl)methylphenylsilan (18): $6,80 \mathrm{~g}$ $(0,28 \mathrm{~mol})$ Magnesium und $0,7 \mathrm{~g} \mathrm{HgCl}_{2}$ in $50 \mathrm{ml}$ THF wurden $30 \mathrm{~min}$ bei Rückflußtemperatur gerührt. Nach Abkühlen auf $50^{\circ} \mathrm{C}$ tropfte man unter weiterem Rühren 3-4 g (ggf. auch weniger) von insgesamt 22,54 $\mathrm{g}(0,28 \mathrm{~mol})$ (Chlormethyl)methylether hinzu. Nach Anspringen der Reaktion wurde sofort auf $-30^{\circ} \mathrm{C}$ abgekühlt und dann bei -30 bis $-25^{\circ} \mathrm{C}$ eine Lösung von $32,90 \mathrm{~g}(0,21 \mathrm{~mol}) 17$ und dem Rest des (Chlormethyl)methylethers in $200 \mathrm{ml}$ THF hinzugetropft. Nach vollständiger Zugabe rührte man $30 \mathrm{~min}$ bei $-30^{\circ} \mathrm{C}$ weiter und setzte das Rühren noch weitere $15 \mathrm{~h}$ fort, wobei man die Temperatur des Reaktionsgemisches auf $10-15^{\circ} \mathrm{C}$ ansteigen ließ. Man verdampfte die leichtflüchtigen Bestandteile unter vermindertem Druck, versetzte den Rückstand mit $400 \mathrm{ml}$ Petrolether, filtrierte, befreite das Filtrat vom Lösungsmittel und destillierte den Rückstand fraktionierend i. Vak. über eine VigreuxKolonne. Ausb. $18,8 \mathrm{~g}(54 \%)$, Sdp. $70{ }^{\circ} \mathrm{C} / 6$ Torr, $n_{\mathrm{D}}^{20}$ 1,504. - ${ }^{1} \mathrm{H}$-NMR $\left(\mathrm{CCl}_{4}\right): \delta=0,26(\mathrm{~d}, J=3,8 \mathrm{~Hz}$; $\left.3 \mathrm{H}, \mathrm{SiCH}_{3}\right), 3,17\left(\mathrm{~s} ; 3 \mathrm{H}, \mathrm{OCH}_{3}\right), 3,17\left(\delta_{\mathrm{B}}\right)$ und 3,20 $\left(\delta_{\mathrm{A}}\right)$ (AB-Teil des ABX-Systems $\mathrm{Si}\left(\mathrm{H}_{\mathrm{X}}\right) \mathrm{CH}_{\mathrm{A}} \underline{\mathrm{H}}_{\mathrm{B}} \mathrm{O}$, $J_{\mathrm{AB}}=-12,9 \mathrm{~Hz}, J_{\mathrm{AX}}=2,1 \mathrm{~Hz}, J_{\mathrm{BX}}=3,0 \mathrm{~Hz} ; 2 \mathrm{H}$, $\mathrm{SiCH}_{2} \mathrm{O}$ ), 4,30 (Zentrum eines $\mathrm{m} ; 1 \mathrm{H}, \mathrm{SiH}$ ), 7,1-7,4 (m; $\left.5 \mathrm{H}, \mathrm{SiC}_{6} \mathrm{H}_{5}\right)$. - IR (Film): $2125 \mathrm{~cm}^{-1}$ $(\mathrm{Si}-\mathrm{H}) .-\mathrm{MS}: m / e=165\left(9 \%, \mathrm{M}^{+}-\mathrm{H}\right), 121(100 \%$, $\mathrm{M}^{+}-\mathrm{CH}_{2} \mathrm{OCH}_{3}$ ).

\section{$\mathrm{C}_{9} \mathrm{H}_{14} \mathrm{OSi}(166,3)$ \\ Ber. C 65,00 H 8,49 Si 16,89, \\ Gef. C 65,1 H 8,5 Si 17,2.}

Als Nebenprodukt der Darstellung von 18 wurden 6,2 $\mathrm{g}$ (Ausb. 14\%, bezogen auf eingesetztes 17) Bis[methoxymethyl]methylphenylsilan isoliert, Sdp. $102{ }^{\circ} \mathrm{C} / 5$ Torr, $n_{\mathrm{D}}^{20} 1,500$. $-{ }^{1} \mathrm{H}-\mathrm{NMR}\left(\mathrm{CCl}_{4}\right): \delta=$ $0,25\left(\mathrm{~s} ; 3 \mathrm{H}, \mathrm{SiCH}_{3}\right), 3,22\left(\mathrm{~s} ; 6 \mathrm{H}, \mathrm{OCH}_{3}\right), 3,22\left(\delta_{\mathrm{B}}\right)$ und $3,25\left(\delta_{\mathrm{A}}\right)\left(\mathrm{AB}-\right.$ System, $J_{\mathrm{AB}}=-12,9 \mathrm{~Hz} ; 4 \mathrm{H}$,
$\left.\mathrm{SiCH}_{2} \mathrm{O}\right), 7,1-7,4\left(\mathrm{~m} ; 5 \mathrm{H}, \mathrm{SiC}_{6} \mathrm{H}_{5}\right) .-\mathrm{MS}: m / e=$ $210\left(1 \%, \mathrm{M}^{+}\right), 165\left(\mathrm{M}^{+}-\mathrm{CH}_{2} \mathrm{OCH}_{3}\right)$.

$$
\begin{array}{rlll}
\mathrm{C}_{I I} \mathrm{H}_{18} \mathrm{O}_{2} \mathrm{Si} & (210,3) \\
\text { Ber. } & \mathrm{C} 62,81 & \text { H } 8,62 & \text { Si } 13,35, \\
\text { Gef. } & \mathrm{C} 62,1 & \text { H } 8,7 & \text { Si } 13,5 .
\end{array}
$$

Untersuchungen zur thermischen Stabilität von 11, 15, 16 und 18: Es wurden Proben von jeweils 60-80 mg Substanz in NMR-Röhrchen eingeschmolzen und $6 \mathrm{~h}$ auf $200^{\circ} \mathrm{C}$ erhitzt. Mit Ausnahme von 15 ließen sich ${ }^{1}$ H-NMR-spektroskopisch keine Veränderungen feststellen. - $\mathbf{1 5}$ wurde der gleichen Operation auch bei $135^{\circ} \mathrm{C}$ unterworfen; hierbei traten ebenfalls Zersetzungserscheinungen auf, jedoch in geringerem Maße als bei $200{ }^{\circ} \mathrm{C}$. Die Zersetzungsprodukte wurden nicht näher charakterisiert.

Kinetische Untersuchungen zur thermisch induzierten Umwandlung von 6-8: 6 und 7 wurden in Mengen von jeweils $60-80 \mathrm{mg}, \mathbf{8}$ in Form von $0,4-0,5 \mathrm{M}$ Lösungen in $\mathrm{C}_{6} \mathrm{D}_{6}$ unter Feuchtigkeitsausschlu $\beta$ in NMR-Röhrchen eingeschmolzen. Jeweils 10 Proben von jeder Verbindung wurden unterschiedlich lange auf $135(\mathbf{6 , 7})$ bzw. $100^{\circ} \mathrm{C}(\mathbf{8})$ erhitzt, wobei - je nach Substanzprobe - ein Umsatz im Bereich von 20 (Minimalumsatz) bis $80 \%$ (Maximalumsatz) realisiert wurde. Die Proben wurden auf Raumtemp. abgeschreckt und ${ }^{1}$ H-NMR-spektroskopisch vermessen; durch Integration der charakteristischen Signale der Edukte und Produkte wurde der Umsatz $U$ [\%] für jede Probe ermittelt und hieraus unter Einbeziehung der Reaktionszeit $t$ entsprechend $t_{1 / 2}=t \lg 2[2-\lg (100-\mathrm{U})]^{-1}$ die Halbwertszeit $t_{1 / 2}$ berechnet. Dies geschah für alle Einzelmessungen mit anschließender Bildung des arithmetischen Mittels. Diese Mittelwerte von $t_{1 / 2}$ (Abweichung der Einzelwerte maximal 7\%) sind in Tab. I - zusammen mit den hieraus berechneten Werten für die Geschwindigkeitskonstanten $k-$ zusammengestellt.

Der Deutschen Forschungsgemeinschaft und dem Fonds der Chemischen Industrie danken wir für die finanzielle Unterstützung dieser Arbeit. Herrn Priv.Doz. Dr. L. Ernst, GBF, Braunschweig-Stöckheim, gilt unser Dank für die Analyse einiger NMR-Spektren.
[1] R. Tacke und H. Lange, Chem. Ber. 116, 3685 (1983).

[2] R. Tacke, M. Link und H. Zilch, Chem. Ber., im Druck.

[3] R. Tacke, H. Lange und A. Bentlage, Chem. Ber. 115, 3673 (1982).
[4] V. F. Mironov, J. Organomet. Chem. Library 9, 375 (1980).

[5] V. D. Sheludyakov und V. F. Mironov, Russ. Chem. Rev. 46, 1167 (1977). 
[6] Y. Yamamoto und D. S. Tarbell, J. Org. Chem. 36, 2954 (1971).

[7] H. Breederveld, Rec. Trav. Chim. Pays-Bas 81, 276 (1962).

[8] V. D. Sheludyakov, A. D. Kirilin und V. F. Mironov, Zh. Obshch. Khim. 47, 1515 (1977); C. A. 87, 201638 j (1977).

[9] R. Tacke und H. Lange, unveröffentlichte Ergebnisse; H. Lange, Dissertation, Technische Universität Braunschweig 1983.
[10] H. Günther, NMR-Spektroskopie, 2. Aufl., S. 233-236, Georg Thieme Verlag, Stuttgart/New York 1983.

[11] A. E. Lemire und J. C. Thompson, J. Am. Chem. Soc. 93, 1163 (1971).

[12] C. H. Yoder, A. Komoriya, J. E. Kochanowski und F. H. Suydam, J. Am. Chem. Soc. 93, 6515 (1971).

[13] R. Tacke, H. Lange und M. T. Attar-Bashi, Liebigs Ann. Chem. 1982, 1946.

[14] R. Tacke und U. Wannagat, Monatsh. Chem. 106, 1005 (1975). 